Mathematical Research Letters 5, 31-46 (1998)

\title{
MONOMIAL RESOLUTIONS
}

\author{
Dave Bayer, Irena Peeva, and Bernd Sturmfels
}

\section{Introduction}

Let $M$ be a monomial ideal in the polynomial ring $S=k\left[x_{1}, \ldots, x_{n}\right]$ over a field $k$. We are interested in the problem, first posed by Kaplansky in the early 1960 's, of finding a minimal free resolution of $S / M$ over $S$. The difficulty of this problem is reflected in the fact that the homology of arbitrary simplicial complexes can be encoded (via the Stanley-Reisner correspondence [BH,Ho,St]) into the multigraded Betti numbers of $S / M$. In particular, the minimal free resolution may depend on the characteristic of $k$.

We introduce a method for resolving $S / M$ by encoding the entire resolution into a single simplicial complex (Construction 2.1). This method is different from the way simplicial complexes are used in the Stanley-Reisner correspondence to compute Betti numbers.

One of the central ideas in this paper is that there exists a genericity condition, which ensures simply structured homological behavior. The same idea is developed further for toric varieties in [PS]. We call a monomial ideal $M$ generic if no variable $x_{i}$ appears with the same nonzero exponent in two distinct minimal generators of $M$. Almost all monomial ideals are generic, in the sense that those which fail to be generic lie on finitely many hyperplanes in the matrix space of exponents. We prove that the minimal free resolution for any generic monomial ideal $M$ comes (in the sense of Construction 2.1) from a simplicial complex $\Delta_{M}$, which we call the Scarf complex of $M$. If $M$ is Artinian, then the Scarf complex is a regular triangulation of a simplex (Corollary 5.5). In general it need not be pure (Example 3.4), and it need not be shellable (Example 5.2), but it is always contractible (Theorem 5.1). We also relate the Scarf complex to the irreducible decomposition of $M$ and to the Cohen-Macaulay property (Theorem 3.7 and Corollary 3.9).

In the literature we found only two general constructions for resolving arbitrary monomial ideals: Taylor's resolution (cf. [Ei, 17.11]) and Lyubeznik's subcomplex (see [Ly]). For a large number of generators these resolutions are very far from minimal and inefficient for applications. In Section 4 we obtain a nonminimal free resolution which comes from Construction 2.1 and has length at

Received September 15, 1997.

All authors are partially supported by the National Science Foundation. The second and third authors are partially supported by the David and Lucile Packard Foundation. 
most the number of variables, so it is much smaller than Taylor's. Our construction is based on deformation of exponents: we deform $M$ to a nearby generic monomial ideal $M^{\prime}$ by using monomials with real exponents, we compute the Scarf complex $\Delta_{M^{\prime}}$ of the generic ideal $M^{\prime}$, and then we label the vertices of $\Delta_{M^{\prime}}$ with the generators of the original ideal $M$. This defines a nonminimal resolution for $S / M$.

In Section 5 we show that, for generic $M$, the Scarf complex $\Delta_{M}$ appears naturally in the boundary of a certain polytope $P_{M}$. As an application, in Section 6 we show that the Betti numbers of an arbitrary monomial ideal satisfy the inequalities of the Upper Bound Theorem for Convex Polytopes. Even sharper bounds can be derived by relating the Scarf complex $\Delta_{M}$ to the concept of order dimension for partially ordered sets (Theorem 6.4).

\section{Taylor complexes}

Let $M=\left\langle m_{1}, \ldots, m_{r}\right\rangle$ be a monomial ideal in $S=k\left[x_{1}, \ldots, x_{n}\right]$. For each subset $I$ of $\{1, \ldots, r\}$ we set $m_{I}:=\operatorname{lcm}\left(m_{i} \mid i \in I\right)$. Let $a_{I} \in \mathbb{N}^{n}$ be the exponent vector of $m_{I}$ and let $S\left(-a_{I}\right)$ be the free $S$-module with one generator in multidegree $a_{I}$. The Taylor resolution of $S / M$ is the $\mathbb{Z}^{n}$-graded module $\mathbb{F}=$ $\bigoplus_{I \subseteq\{1, \ldots, r\}} S\left(-a_{I}\right)$ with basis denoted by $\left\{e_{I}\right\}_{I \subseteq\{1, \ldots, r\}}$ and equipped with the differential

$$
d\left(e_{I}\right)=\sum_{i \in I} \operatorname{sign}(i, I) \cdot \frac{m_{I}}{m_{I \backslash i}} \cdot e_{I \backslash i}
$$

where $\operatorname{sign}(i, I)$ is $(-1)^{j+1}$ if $i$ is the $j$ th element in the ordering of $I$. This is a free resolution of $S / M$ over $S$ having length $r$ and $2^{r}$ terms. It is very far from minimal if $r \gg n$. A smaller resolution based on Taylor's resolution was constructed in $[\mathrm{Ly}]$.

Every simplicial complex $\Delta$ on $\{1, \ldots, r\}$ defines a submodule $\mathbb{F}_{\Delta}:=$ $\bigoplus_{I \in \Delta} S\left(-a_{I}\right)$ of the Taylor resolution $\mathbb{F}$ which is closed under the differential (2.1). This $\mathbb{N}^{n}$-graded complex of free $S$-modules is described in more detail in the next construction.

Construction 2.1. (Monomial resolution from a labeled simplicial complex)

Let $\Delta$ be a simplicial complex whose vertices are labeled by the generators of $M$. We label each face of $\Delta$ by the least common multiple of its vertices. The exponent vectors of these monomials define an $\mathbb{N}^{n}$-grading of $\Delta$. Let $\mathbb{F}_{\Delta}$ be the $\mathbb{N}^{n}$-graded chain complex of $\Delta$ over $S$. It is obtained from the simplicial chain complex by homogenizing the differential. For example, if $m_{1}=x^{3} y^{4}$ and $m_{2}=x z^{3}$ form an edge, then its boundary is $d\left(e_{12}\right)=z^{3} e_{1}-x^{2} y^{4} e_{2}$. If the complex $\mathbb{F}_{\Delta}$ is exact then we call it the resolution defined by the labeled simplicial complex $\Delta$. Such a resolution is characteristic-free and an associative commutative differential graded algebra. In this case the $\mathbb{N}^{n}$-graded Hilbert series of $S / M$ equals the $\mathbb{N}^{n}$-graded Euler characteristic of $\Delta$ divided by (1$\left.x_{1}\right) \cdots\left(1-x_{n}\right)$.

We use reduced simplicial homology to determine when $\mathbb{F}_{\Delta}$ is exact. 
Lemma 2.2. The complex $\mathbb{F}_{\Delta}$ is exact if and only if for every monomial $m$ the simplicial complex $\Delta[m]=\left\{I \in \Delta \mid m_{I}\right.$ divides $\left.m\right\}$ is empty or acyclic over $k$.

Proof. Since $\mathbb{F}_{\Delta}$ is $\mathbb{N}^{n}$-graded it suffices to check exactness in each multidegree. The component of $\mathbb{F}_{\Delta}$ in multidegree $m$ is a complex of finite-dimensional $k$ vector spaces, which can be identified with the chain complex of $\Delta[m]$ over $k$.

Remark 2.3. Lemma 2.2 shows that Taylor's complex $\mathbb{F}$ is exact. In this case $\Delta$ is a full $(r-1)$-simplex and $\Delta[m]$ is the subsimplex on the minimal generators which divide $m$.

\section{Generic monomial ideals}

For any monomial ideal $M=\left\langle m_{1}, \ldots, m_{r}\right\rangle$ we define a simplicial complex:

$$
\begin{aligned}
\Delta_{M} & := \\
& \left\{I \subseteq\{1, \ldots, r\} \mid m_{I} \neq m_{J} \text { for all } J \subseteq\{1, \ldots, r\} \text { other than } I\right\}
\end{aligned}
$$

In Section 5 we will show that $\Delta_{M}$ equals a complex introduced by Herbert Scarf in the context of mathematical economics (see [Sc, §2.8]). We call $\Delta_{M}$ the Scarf complex of $M$.

Taylor's resolution $\mathbb{F}$ is a direct sum of the minimal free resolution of $S / M$ and trivial complexes $0 \longrightarrow S\left(-a_{I}\right) \longrightarrow S\left(-a_{I}\right) \longrightarrow 0$. On the other hand, if $I \in \Delta_{M}$ then $\mathbb{F}$ has a unique minimal generator in multidegree $a_{I}$. Therefore, the minimal free resolution of $S / M$ always contains the complex $\mathbb{F}_{\Delta_{M}}$, but is larger in general. More precisely, for every monomial ideal $M$ and every face $I$ of $\Delta_{M}$, the minimal free resolution of $S / M$ has a unique generator in multidegree $a_{I}$ and the differential acts on these generators as in (2.1).

Lemma 3.1. If all nonzero Betti numbers of $S / M$ are concentrated in the multidegrees $a_{I}$ of the faces $I$ of $\Delta_{M}$, then $\mathbb{F}_{\Delta_{M}}$ is the minimal free resolution of $S / M$.

Proof. If the minimal free resolution of $S / M$ is strictly larger than $\mathbb{F}_{\Delta_{M}}$, then the Taylor resolution has at least two basis elements in some multidegree $a_{I}$ for $I \in \Delta_{M}$. This contradicts the definition of $\Delta_{M}$.

Theorem 3.2. Let $M$ be a generic monomial ideal. Then the complex $\mathbb{F}_{\Delta_{M}}$ defined by the Scarf complex $\Delta_{M}$ is the minimal free resolution of $S / M$ over $S$.

Proof. If $I \in \Delta_{M}$ and $i \in I$ then $m_{I \backslash i}$ properly divides $m_{I}$. Thus we see directly from (2.1) that $\mathbb{F}_{\Delta_{M}}$ is minimal. It remains to show that $\mathbb{F}_{\Delta_{M}}$ is exact. We will apply Lemma 3.1. Consider any multidegree $a_{I}$ with $I \notin \Delta_{M}$. The $j$ th Betti number in multidegree $a_{I}$ equals the $k$-dimension of the homology of the Koszul complex at $\mathbb{K}_{j}:=\wedge^{j}(S / M)^{n}$ in degree $a_{I}$. The component of $\mathbb{K}_{j}$ in degree $a_{I}$ is contained in the $S / M$-module $\frac{m_{I}}{\operatorname{supp}\left(m_{I}\right)} \mathbb{K}_{j}$, where $\operatorname{supp}\left(m_{I}\right)$ is the maximal square-free monomial dividing $m_{I}$. To prove that this component is 
zero, it suffices to show that $\frac{m_{I}}{\operatorname{supp}\left(m_{I}\right)}$ is zero in $S / M$. Choosing $I$ minimal with respect to inclusion, we may assume $m_{I}=m_{I \cup i}$ for some $i \in\{1, \ldots, r\} \backslash I$. The monomials $m_{i}$ and $m_{I}$ have different exponents in any fixed variable because $M$ is generic. So $m_{i}$ divides $\frac{m_{I}}{\operatorname{supp}\left(m_{I}\right)}$, and we are done.

Corollary 3.3. Let $M$ be a generic monomial ideal.

(1) The number of $j$-faces of the Scarf complex $\Delta_{M}$ equals the total Betti number $\beta_{j+1}(S / M)=\operatorname{dim}_{k} \operatorname{Tor}_{j+1}^{S}(S / M, k)$.

(2) The minimal free resolution of $S / M$ is characteristic free. It is $\mathbb{N}^{n}$-graded and in each multidegree the Betti number is either 0 or 1.

(3) The $\mathbb{N}^{n}$-graded Hilbert series of $S / M$ (i.e. the sum of all monomials not in $M$ ) is

$$
\frac{\sum_{I \in \Delta_{M}}(-1)^{|I|} \cdot m_{I}}{\left(1-x_{1}\right) \cdots\left(1-x_{n}\right)}
$$

and there are no cancellations in the alternating sum in the numerator.

The multigraded Betti numbers of any monomial ideal determine its Hilbert series, but not conversely. For a generic monomial ideal, by Corollary 3.3, the multigraded Hilbert series determines the multigraded Betti numbers, and, by Theorem 3.2, they determine the Scarf complex and the minimal free resolution. Thus for a generic monomial ideal, the multigraded Hilbert series determines the minimal free resolution; in particular, these two are computationally equivalent. We next illustrate our results for a small example.

Example 3.4. Let $n=3, r=4$ and consider the generic monomial ideal $M=\left\langle x^{2} z^{3}, x^{3} z^{2}, x y z, y^{2}\right\rangle$. The Scarf complex $\Delta_{M}$ is a triangle connected to an edge:
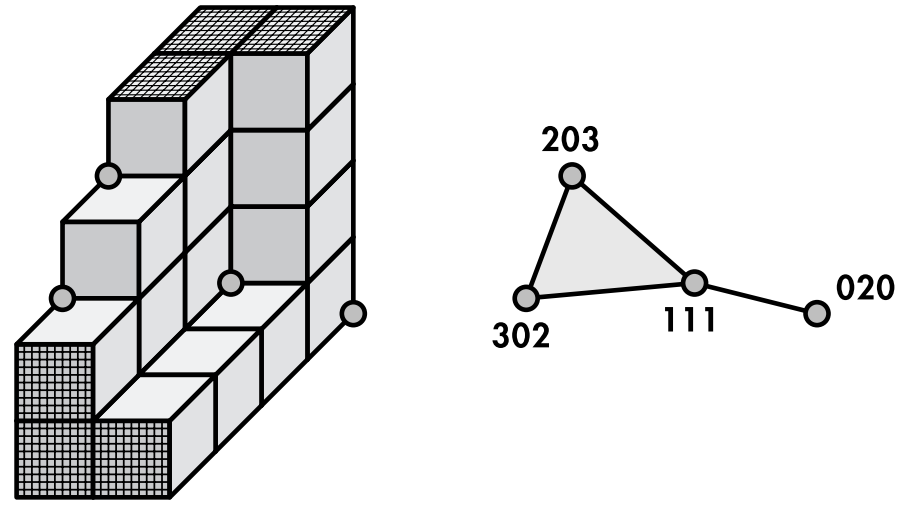

The triangle is labeled by $x^{3} y z^{3}$, the edges of the triangle are labeled by $x^{3} z^{3}$, $x^{2} y z^{3}, x^{3} y z^{2}$, and the other edge by $x y^{2} z$. The minimal free resolution of $S / M$ is the $\mathbb{N}^{3}$-graded chain complex of the simplicial complex depicted above: 


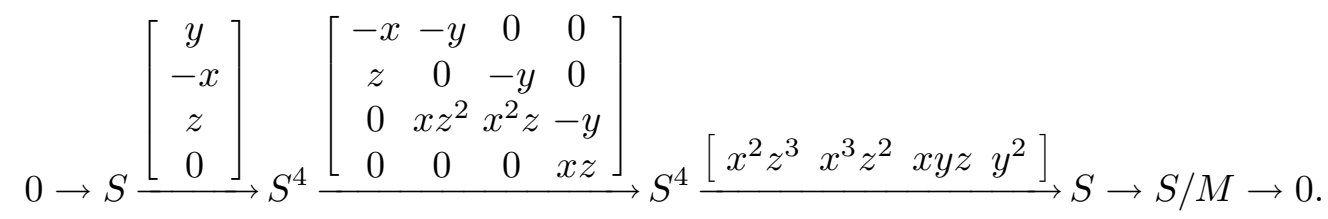

The Hilbert series of $S / M$ equals

$$
\begin{gathered}
\sum\left\{x^{a} y^{b} z^{c}: x^{a} y^{b} z^{c} \notin M\right\}=\frac{P(x, y, z)}{(1-x)(1-y)(1-z)}, \quad \text { where } \\
P(x, y, z)=1-x^{2} z^{3}-x^{3} z^{2}-x y z-y^{2}+x^{3} z^{3}+x^{2} y z^{3}+x^{3} y z^{2}+x y^{2} z-x^{3} y z^{3} .
\end{gathered}
$$

This polynomial is the $\mathbb{N}^{3}$-graded Euler characteristic of the Scarf complex.

A resolution $F$ of a cyclic module $S / L$ is a $D G$-algebra if it admits an associative, graded (by homological degree), skew commutative product $*$ satisfying the Leibnitz rule $d(\alpha * \beta)=d(\alpha) * \beta+(-1)^{\operatorname{deg}(\alpha)} \alpha * d(\beta)$. The prototypical example of a DG-algebra is a Koszul complex, with product given by exterior multiplication. See $[\mathrm{Ku}]$ for motivation and further examples, where quotient DG-algebras are also considered. The minimal free resolution of a monomial ideal need not be a DG-algebra:

Example 3.5. (Avramov, Backelin) There is no DG-algebra structure on the minimal free resolution of $S /\left\langle x^{2}, x y^{2} z, y^{2} z^{2}, y z^{2} w, w^{2}\right\rangle$. This is proved in $[\mathrm{Av}, 5.2 .3]$.

On the other hand, the Taylor resolution of a monomial ideal is always a DG-algebra, and Theorem 3.2 has the following corollary:

Corollary 3.6. If $M$ is a generic monomial ideal, then the minimal free resolution of $S / M$ is a quotient DG-algebra of the Taylor resolution.

Proof. We prove that any complex $F_{\Delta}$ given by Construction 2.1 is a quotient DG-algebra of the Taylor resolution. The Taylor resolution $F$ can be understood as a divided Koszul complex, and a product modeled after exterior multiplication gives it a DG-algebra structure: Define

$$
e_{I} * e_{J}:=\left\{\begin{array}{cl}
\operatorname{sign}(I, J) \cdot \frac{m_{I} m_{J}}{m_{I \cup J}} \cdot e_{I \cup J} & \text { if } I \cap J=\emptyset, \\
0 & \text { otherwise. }
\end{array}\right.
$$

Here, if $I=\left\{i_{1}<\ldots<i_{q}\right\}$ and $J=\left\{j_{1}<\ldots<j_{p}\right\}$, then $\operatorname{sign}(I, J)$ is the sign of the permutation which makes $i_{1}, \ldots, i_{q}, j_{1}, \ldots, j_{p}$ an increasing sequence. This product is multigraded, and like exterior multiplication it is associative, skew commutative, and satisfies Leibniz's rule. For a simplicial complex $\Delta$ the kernel of the map $F \rightarrow F_{\Delta}$ is a DG-ideal of $F$, so $F_{\Delta}$ is a quotient DG-algebra of $F$. 
In the rest of this section we describe the irreducible decomposition of a generic monomial ideal $M$, that is, the unique minimal expression of $M$ as an intersection of ideals of the form $\left\langle x_{i_{1}}^{d_{1}}, x_{i_{2}}^{d_{2}}, \ldots, x_{i_{j}}^{d_{j}}\right\rangle$. Choose an integer $D$ larger than the degree of any minimal generator of $M$. We replace $M$ by the Artinian ideal

$$
M^{*}:=M+\left\langle x_{1}^{D}, x_{2}^{D}, \ldots, x_{n}^{D}\right\rangle .
$$

Let $\Delta_{M^{*}}$ be the Scarf complex of $M^{*}$. This is a simplicial complex on $\{1,2, \ldots, r, r+1, \ldots, r+n\}$, where the index $r+i$ is associated with the generator $x_{i}^{D}$. By Corollary 5.5 below, $\Delta_{M^{*}}$ pure $(n-1)$-dimensional. For each facet $I$ of $\Delta_{M *}$ we form the irreducible ideal

$$
M_{I}:=\left\langle x_{s}^{p_{s}}: p_{s}=\operatorname{deg}_{x_{s}}\left(m_{I}\right) \text { and } p_{s}<D\right\rangle .
$$

Note that $M_{I}$ is independent of the choice of $D$ and may have less than $n$ generators.

Theorem 3.7. A generic monomial ideal $M$ is the intersection of the irreducible ideals $M_{I}$, where I runs over all facets of the Scarf complex $\Delta_{M^{*}}$. This intersection is irredundant.

Proof. We first show that $M$ is contained in $M_{I}$ for every facet $I$ of $\Delta_{M^{*}}$. Let $m_{j}$ be any minimal generator of $M$. If $j \in I$ then there exists a variable $x_{s}$ with $\operatorname{deg}_{x_{s}}\left(m_{I}\right)=\operatorname{deg}_{x_{s}}\left(m_{j}\right)<D$. This implies $m_{j} \in M_{I}$. If $j \notin I$ then there is a variable $x_{s}$ with $\operatorname{deg}_{x_{s}}\left(m_{I}\right)<\operatorname{deg}_{x_{s}}\left(m_{j}\right)<D$, which implies $m_{j} \in M_{I}$ as well.

For the reverse inclusion $\bigcap_{I} M_{I} \subseteq M$, we shall prove that every monomial $m$, which is not in $M$ is not in $M_{I}$ for some facet $I$ of $\Delta_{M^{*}}$. Let $m \notin M$. We choose a $D \gg 0$ so that $m \notin M^{*}$. Next we select a monomial $\tilde{m}$ such that $m \cdot \tilde{m} \notin M^{*}$ but $x_{i} \cdot m \cdot \tilde{m} \in M^{*}$ for $i=1,2, \ldots, n$. There exist unique (and necessarily distinct) minimal generators $m_{j_{1}}, \ldots, m_{j_{n}}$ of $M^{*}$ with the property that $m_{j_{i}}$ divides $x_{i} \cdot m \cdot \tilde{m}$. Setting $I:=\left\{j_{1}, \ldots, j_{n}\right\}$, we have $x_{1} x_{2} \cdots x_{n} \cdot m \cdot \tilde{m}=m_{I}$. This implies $m \notin M_{I}$.

Finally, we must show that the intersection $\cap M_{I}$ over all facets $I$ of $\Delta_{M^{*}}$ is irredundant. Fix a facet $I$ and consider the monomial $m:=m_{I} /\left(x_{1} x_{2} \cdots x_{n}\right)$. Clearly, $m \notin M_{I}$. It suffices to show that $m \in M_{J}$ for all other facets $J$ of $\Delta_{M^{*}}$. Fix another facet $J$. There exists an index $i \in I \backslash J$ and a variable $x_{s}$ such that $\operatorname{deg}_{x_{s}}\left(m_{I}\right) \geq \operatorname{deg}_{x_{s}}\left(m_{i}\right)>\operatorname{deg}_{x_{s}}\left(m_{J}\right)$. This implies $D \geq \operatorname{deg}_{x_{s}}\left(m_{I}\right)>$ $\operatorname{deg}_{x_{s}}(m) \geq \operatorname{deg}_{x_{s}}\left(m_{J}\right)$ and therefore $m \in M_{J}$.

Example 3.8. The seven irreducible components of the generic monomial ideal $\left\langle x y^{2} z^{3}, x^{3} y z^{2}, x^{2} y^{3} z\right\rangle=\langle x\rangle \cap\langle y\rangle \cap\langle z\rangle \cap\left\langle x^{3}, y^{2}\right\rangle \cap\left\langle y^{3}, z^{2}\right\rangle \cap\left\langle z^{3}, x^{2}\right\rangle \cap\left\langle x^{3}, y^{3}, z^{3}\right\rangle$

correspond to the seven triangles in the Scarf complex of the Artinian ideal $\left\langle x^{4}, y^{4}, z^{4}, x y^{2} z^{3}, x^{3} y z^{2}, x^{2} y^{3} z\right\rangle$. This Scarf complex is depicted in Example 5.6 . 
Corollary 3.9. Let $M$ be a generic monomial ideal and $M=\cap_{I \in \Delta_{M^{*}}} M_{I}$ its irreducible irredundant decomposition. Then

$$
\operatorname{depth}(S / M)=\min _{I \in \Delta_{M^{*}}}\left\{\operatorname{dim}\left(M_{I}\right)\right\}
$$

In particular, $M$ is Cohen-Macaulay if and only if it is pure-dimensional.

Proof. Each facet $I$ of $\Delta_{M^{*}}$ defines a component $M_{I}$ with

$$
\operatorname{dim}\left(M_{I}\right)=|I \cap\{r+1, \ldots, r+n\}| .
$$

To compute the depth of $S / M$ we consider the Scarf complex $\Delta_{M}$. Note that $\Delta_{M}$ is a subcomplex of $\Delta_{M^{*}}$ which may have dimension less than $n-1$ and is generally not pure. The depth of $S / M$ equals the minimum of the numbers

$n-|J|$ where $J$ runs over all facets of $\Delta_{M}$. Every facet $J$ of $\Delta_{M}$ extends to a facet $I$ of $\Delta_{M^{*}}$, and, conversely, if $I$ is a facet of $\Delta_{M^{*}}$ then $I \cap\{1, \ldots, n\}$ is a face of $\Delta_{M}$. Therefore

$$
\operatorname{depth}(S / M)=\min \left\{|I \cap\{r+1, \ldots, r+n\}|: I \text { facet of } \Delta_{M^{*}}\right\}
$$

The ideal $M$ being pure-dimensional means that all irreducible components $M_{I}$ have the same dimension. So $M$ is pure-dimensional if and only if $\operatorname{dim}(M)=$ $\operatorname{depth}(S / M)$.

\section{Deformation of exponents}

Let $M=\left\langle m_{1}, \ldots, m_{r}\right\rangle$ be an arbitrary monomial ideal; say, $M$ is not generic. In this section we construct a (typically nonminimal) free resolution of $S / M$ by deforming the exponent vectors of the generators of $M$. This approach has the following advantages:

- The resolution by deformation of exponents has length at most the number of variables; thus in general it is much smaller and shorter than Taylor's resolution.

- This resolution is a DG-algebra (by the proof of Corollary 3.6).

- Section 5 relates the Betti numbers to the $f$-vector of a polytope $P_{M}$.

Construction 4.1. Let $\left\{a_{i}=\left(a_{i 1}, \ldots, a_{i n}\right) \mid 1 \leq i \leq r\right\}$ be the exponent vectors of the minimal generators of $M$. Choose vectors $\epsilon_{i}=\left(\epsilon_{i 1}, \ldots, \epsilon_{i n}\right) \in \mathbb{R}^{n}$ for $1 \leq i \leq r$ such that, for all $i$ and all $s \neq t$, the numbers $a_{i s}+\epsilon_{i s}$ and $a_{i t}+\epsilon_{i t}$ are distinct, and

$$
a_{i s}+\epsilon_{i s}<a_{i t}+\epsilon_{i t} \quad \text { implies } \quad a_{i s} \leq a_{i t} .
$$

The last condition is satisfied for all sufficiently small positive $\epsilon_{i}$. Each vector $\epsilon_{i}$ defines a monomial $\mathbf{x}^{\epsilon_{i}}=x_{1}^{\epsilon_{i 1}} \cdots x_{n}^{\epsilon_{i n}}$ with real exponents. Abbreviate $\epsilon:=$ 
$\left(\epsilon_{1}, \ldots, \epsilon_{r}\right)$. We formally introduce the generic monomial ideal (in a polynomial ring with real exponents):

$$
M_{\epsilon}:=\left\langle m_{1} \cdot \mathbf{x}^{\epsilon_{1}}, m_{2} \cdot \mathbf{x}^{\epsilon_{2}}, \ldots, m_{r} \cdot \mathbf{x}^{\epsilon_{r}}\right\rangle .
$$

We call $M_{\epsilon}$ a generic deformation of $M$. Let $\Delta_{M_{\epsilon}}$ be the Scarf complex of $M_{\epsilon}$. We now label the vertex of $\Delta_{M_{\epsilon}}$ corresponding to $m_{i} \cdot \mathbf{x}^{\epsilon_{i}}$ with the original monomial $m_{i}$. Let $\mathbb{F}_{\epsilon}$ be the complex of $S$-modules defined by this labeling of $\Delta_{M_{\epsilon}}$ as in Construction 2.1.

Example 4.2. A simple way of deforming $M$ is to pick an integer $\nu>r$ and set $M_{\epsilon}:=\left\langle m_{i}^{\nu} \cdot\left(x_{1} x_{2} \cdots x_{n}\right)^{i}: i=1, \ldots, r\right\rangle$. This amounts to choosing $\epsilon_{i j}=i / \nu$ in Construction 4.1 since the ideals $M_{\epsilon}$ and $\left\langle m_{i} \cdot\left(x_{1} x_{2} \cdots x_{n}\right)^{\frac{i}{\nu}}: i=1, \ldots, r\right\rangle$ have the same Scarf complex. If $M$ is square-free then $\left\langle m_{1}, m_{2}^{2}, \ldots, m_{r}^{r}\right\rangle$ is a generic deformation of $M$.

Theorem 4.3. The complex $\mathbb{F}_{\epsilon}$ is a free resolution of $S / M$ over $S$.

Proof. Fix a monomial $m$. Let $J$ be the largest subset of $\{1, \ldots, r\}$ such that $m_{J}$ divides $m$. The following conditions are equivalent for a subset $I$ of $\{1, \ldots, r\}$ :

$m_{I}$ divides $m \Longleftrightarrow I \subseteq J \Longleftrightarrow m_{I}$ divides $m_{J} \Longleftrightarrow m_{I}(\epsilon)$ divides $m_{J}(\epsilon)$.

Here $m_{I}(\epsilon):=\operatorname{lcm}\left(m_{i} \mathbf{x}^{\epsilon_{i}}: i \in I\right)$. The last equivalence follows from our choice of the $\epsilon_{i j}$. The set of all faces of $\Delta_{M_{\epsilon}}$ which satisfy the four equivalent conditions above is an acyclic simplicial complex, by Theorem 3.2 and Lemma 2.2 applied to $M_{\epsilon}\left[m_{J}(\epsilon)\right]$. Now apply Lemma 2.2 to $M$ and $m$ with $\Delta=\Delta_{M_{\epsilon}}$.

Corollary 4.4. The Betti numbers of $M$ are less than or equal to those of any deformation $M_{\epsilon}$, that is, less than or equal to the face numbers of the Scarf complex $\Delta_{M_{\epsilon}}$.

We emphasize that the Betti numbers of $M_{\epsilon}$ depend on the choice of the generic deformation. There are finitely many complexes $\Delta_{M_{\epsilon}}$ which can be obtained by Construction 4.1 and each of them corresponds to an $\epsilon$ lying in an open convex polyhedral cone in $\mathbb{R}^{r \cdot n}$.

Example 4.5. Consider the ideal $M=\left\langle x^{2}, x y^{2} z, y^{2} z^{2}, y z^{2} w, w^{2}\right\rangle$ in Example 3.5. A generic deformation is $M_{\epsilon}=\left\langle x^{2}, x y^{2} z, y^{3} z^{3}, y z^{2} w, w^{2}\right\rangle$. Label the generators as $1,2,3,4,5$ in the given order. The Scarf complex of $M_{\epsilon}$ consists of the tetrahedron $\{1,2,4,5\}$ and the triangle $\{2,3,4\}$. Applying Construction 4.1 we obtain a nonminimal free resolution $\mathbb{F}_{\epsilon}$ of $S / M$. The Betti numbers of $S / M$ are $1,5,7,4,1$ while the Betti numbers of $S / M_{\epsilon}$ are $1,5,8,5,1$. Thus $\mathbb{F}_{\epsilon}$ differs from the minimal resolution by a single summand $0 \rightarrow S \rightarrow S \rightarrow 0$, placed in homological degrees 2 and 3. However, this makes a big difference structurally: by Corollary 3.6, the resolution $\mathbb{F}_{\epsilon}$ is a DG-algebra (with a simple multiplication rule) while the minimal free resolution admits no DG-algebra structure at all. 
Note that Taylor's resolution is one step longer than $\mathbb{F}_{\epsilon}$. It has Betti numbers $1,5,10,10,5,1$.

Remarks 4.6. (a) In deforming monomial ideals we are guided by the intuition of continuously varying real exponents. However, no algebra in a polynomial ring with real exponents is used. The Scarf complex of a generic monomial ideal depends only on the coordinatewise order of the exponents of the generators. If the exponents are real numbers, then we can replace them by integers while preserving their order coordinatewise. We will obtain a monomial ideal with integer exponents and the same Scarf complex.

(b) The irreducible decomposition of any monomial ideal $M$ can be computed by applying Theorem 3.7 to a generic deformation $M_{\epsilon}$ and then setting $\epsilon=0$.

(c) Let $W$ be a homogeneous ideal in $S$. If $u$ is a non-zero-divisor in $S / W$ and $\mathbb{G}$ is a resolution of $S / W$ over $S$, then $\mathbb{G} \otimes S /\langle u\rangle$ is a resolution of $S / W+\langle u\rangle$ over $S /\langle u\rangle$. This is sometimes called "deforming the resolution" and is very useful in Gröbner basis theory and for polarizing monomial ideals into squarefree monomial ideals. We emphasize that it is very different from Construction 4.1. When "deforming the resolution", one just sets $u=0$ in the matrices of the differentials in $\mathbb{G}$, while Construction 4.1 changes the entries in these matrices in accordance with the changes of the degrees of the generators.

\section{Convexity}

Polytopes are a powerful tool for structuring combinatorial data appearing in algebra and algebraic geometry. For example, Newton polytopes play a significant role in computer algebra, singularity theory and toric geometry. In this section we present the polytope underlying the minimal free resolution of a generic monomial ideal $M$.

Let $M[m]$ be the subideal of $M$ generated by the generators of $M$ which divide a given monomial $m$. Then $M[m]$ is a generic monomial ideal as well, and its Scarf complex equals

$$
\Delta_{M[m]}=\Delta_{M}[m]
$$

Lemma 2.1 and (5.1) imply that the exactness of $\mathbb{F}_{\Delta_{M}}$ for all $M$ is equivalent to the acyclicity of $\Delta_{M}$ for all $M$. In fact, the following stronger result holds.

Theorem 5.1. The Scarf complex $\Delta_{M}$ of a generic monomial ideal $M$ is contractible.

The Scarf complex need not be pure, and it need not be shellable either:

Example 5.2. Let $M:=\left\langle x y z, x^{4} y^{3}, x^{3} y^{5}, y^{4} z^{3}, y^{2} z^{4}, x^{2} z^{2}\right\rangle$. The Scarf complex of this generic monomial ideal consists of two triangles and an edge meeting at a vertex. 

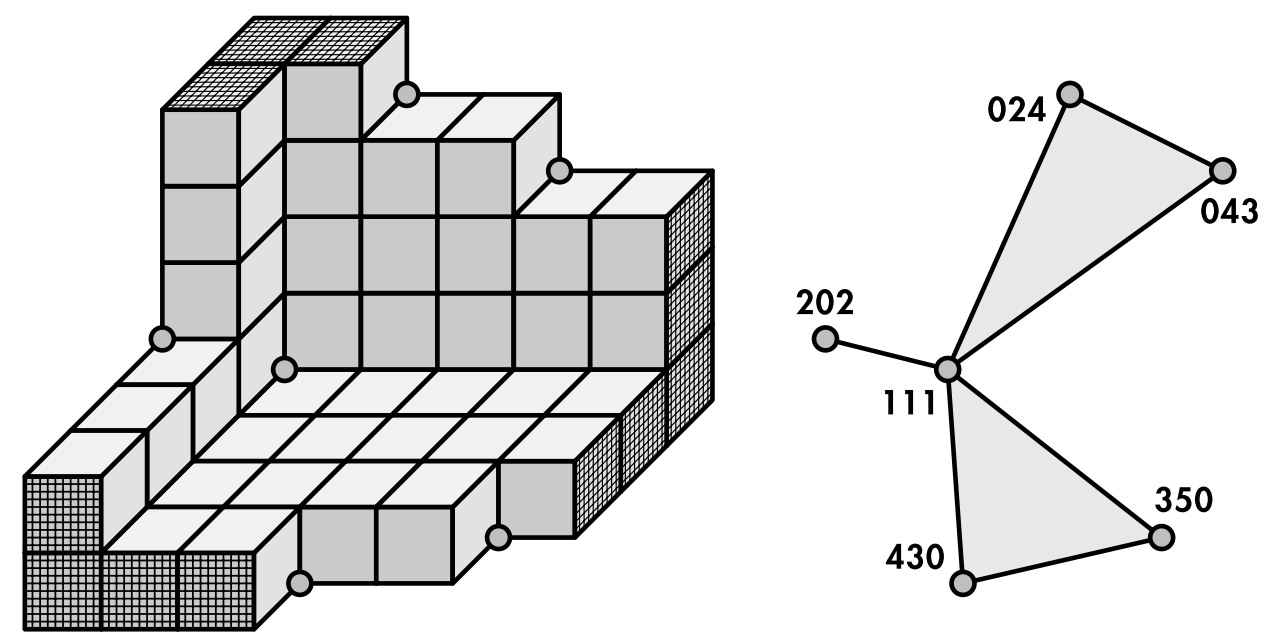

Here $\Delta_{M}$ is contractible, but not shellable, even in the nonpure sense of [BW].

Theorem 5.1 will be derived from Proposition 5.3, which is an extension of Theorem 2.8.4 in [Sc, §2.8]. In Scarf's book the faces of $\Delta_{M}$ are called "primitive sets". Scarf's original definition of "primitive sets" in [Sc, §2.8] is as follows:

$$
I \in \Delta_{M} \quad \Longleftrightarrow \quad \forall i \in\{1, \ldots, r\} \exists j \in\{1, \ldots, n\}: a_{I j} \leq a_{i j}
$$

where $a_{I j}=\max \left\{a_{i j} \mid i \in I\right\}$. We will prove that this is equivalent to our definition (3.1).

Proposition 5.3. Let $M$ be a generic monomial ideal. There exists a polytope $P_{M}$ in $\mathbb{R}^{n}$ such that $\Delta_{M}$ is isomorphic to the subcomplex of the boundary of $P_{M}$ consisting of all faces supported by a strictly positive inner normal vector.

Construction 5.4. [Sc] One possible choice of a polytope $P_{M}$ satisfying Proposition 5.3 is as follows. Let $a_{i}=\left(a_{i 1}, a_{i 2}, \ldots, a_{i n}\right) \in \mathbb{N}^{n}$ be the exponent vector of the $i$-th minimal generator of $M$. We fix a sufficiently large real number $t \gg 0$ and define $P_{M}$ as the convex hull of the point set

$$
\left\{\left(a_{i 1}^{t}, a_{i 2}^{t}, \ldots, a_{i n}^{t}\right) \mid 1 \leq i \leq r\right\} \quad \subset \quad \mathbb{R}^{n} .
$$

The combinatorial type of $P_{M}$ is independent of $t$ for large $t$.

Proof of Proposition 5.3. We identify each face of $P_{M}$ with a subset $I \subseteq$ $\{1, \ldots, r\}$, namely, the indices of vertices which lie on that face. Let $I$ be a face of $P_{M}$ with inner normal vector $\left(w_{1}, \ldots, w_{n}\right)$ where $w_{j}>0$ for $j=1, \ldots, n$. We may assume

$$
\forall i \in\{1, \ldots, r\}: w_{1} \cdot a_{i 1}^{t}+\cdots+w_{n} \cdot a_{i n}^{t} \geq 1,
$$


and equality holds in (5.4) if and only if $i \in I$. This implies $a_{I j} \leq w_{j}^{-1 / t}$ for all $j \in\{1, \ldots, n\}$. At least one of the summands in (5.4) is greater or equal to $1 / n$. Consequently,

$$
\forall i \in\{1, \ldots, r\} \exists j \in\{1, \ldots, n\}: \quad a_{i j} \geq w_{j}^{-1 / t} \cdot n^{-1 / t} \geq a_{I j} \cdot n^{-1 / t} .
$$

Now let $t \rightarrow \infty$. Then we can erase the factor $n^{-1 / t} \rightarrow 1$, and (5.2) is satisfied.

For the converse we consider the special case where $M$ is Artinian. We first show that $\Delta_{M}$ is pure of dimension $n-1$. We may assume that $a_{i j}=0$ for $1 \leq i \neq j \leq n$ and $a_{l i}<a_{i i}$ for $1 \leq i \leq n<l \leq r$. Consider any $I \in \Delta_{M}$ with $|I|<n$. Then there exists $i \in I$ such that $a_{i}$ and $a_{I}$ agree in at least two coordinates, say $j$ and $j^{\prime}$. Consider the set

$$
\mathcal{S}:=\quad\left\{l \in\{1, \ldots, r\} \mid a_{l j}>a_{i j}=a_{I j} \text { and } \forall i \in I \exists s: a_{i s}>a_{l s}\right\} .
$$

It is nonempty since $j \in \mathcal{S}$. Select $l \in \mathcal{S}$ with $a_{l j}$ smallest. Then $I \cup\{l\} \in \Delta_{M}$.

We next consider the oriented matroid [BLSWZ] of the configuration in (5.3) plus the origin. Set $a_{0 j}:=0$ for all $j$ and define for $0 \leq i_{0}<\ldots<i_{n} \leq r$

$$
\left[i_{0}, i_{1}, \ldots, i_{n}\right]:=\operatorname{sign}\left(\operatorname{det}\left(\begin{array}{ccccc}
1 & a_{i_{0} 1}^{t} & a_{i_{0} 2}^{t} & \cdots & a_{i_{0} n}^{t} \\
1 & a_{i_{1} 1}^{t} & a_{i_{1} 2}^{t} & \cdots & a_{i_{1} n}^{t} \\
\vdots & \vdots & \vdots & \ddots & \vdots \\
1 & a_{i_{n} 1}^{t} & a_{i_{n} 2}^{t} & \cdots & a_{i_{n} n}^{t}
\end{array}\right)\right) .
$$

Let $I=\left\{i_{1}, \ldots, i_{n}\right\}$ be a maximal face of $\Delta_{M}$. There exists a unique permutation $\sigma=\left(\sigma_{1}, \ldots, \sigma_{n}\right)$ of $I$ such that $a_{I j}=a_{\sigma_{j} j}$ for all $j$. Since $t \gg 0$, we have

$$
\operatorname{sign}(\sigma)=\left[0, i_{1}, \ldots, i_{n}\right]
$$

For $j \notin I$ there exists an $s$ with $a_{j s}>a_{\sigma_{j} s}$. Laplace expansion along the $s$-th column gives

$$
\left[j, i_{1}, \ldots, i_{n}\right]=-\operatorname{sign}(\sigma) \text { for all } j \notin I .
$$

This shows that $I$ is a facet of $P_{M}$ which is visible from the origin. Since $P_{M}$ intersects each coordinate axis, the normal vector of $I$ is strictly positive.

Now drop the assumption that $M$ is Artinian and let $I$ be a maximal face of $\Delta_{M}$. Let $M^{\prime}$ be an Artinian ideal obtained from $M$ by adding large powers of the variables. Then $I$ lies in $\Delta_{M^{\prime}}$ as well. Therefore $I$ is a face of $P_{M^{\prime}}$ having a positive inner normal vector, and since $P_{M} \subseteq P_{M^{\prime}}$, that same positive vector is minimized over $P_{M}$ at $I$.

It follows from our discussion that the Scarf complex $\Delta_{M}$ is pure and shellable when $M$ is Artinian, that is, when every variable $x_{i}$ appears to some power in $M$. 
Corollary 5.5. If $M$ is Artinian and generic, then $\Delta_{M}$ is a regular triangulation of the $(n-1)$-simplex.

Proof. See [Zi] for the definition and basic properties of regular triangulations. The polytope $P_{M}$ lies in the positive orthant and intersects each coordinate axis. Each face of $P_{M}$ visible from the origin is a simplex. The set of these faces is $\Delta_{M}$.

Example 5.6. Consider the ideal $M=\left\langle x^{4}, y^{4}, z^{4}, x y^{2} z^{3}, x^{3} y z^{2}, x^{2} y^{3} z\right\rangle$. This ideal is Artinian and generic. The Scarf complex $\Delta_{M}$ of $M$ is a regular triangulation of a triangle:

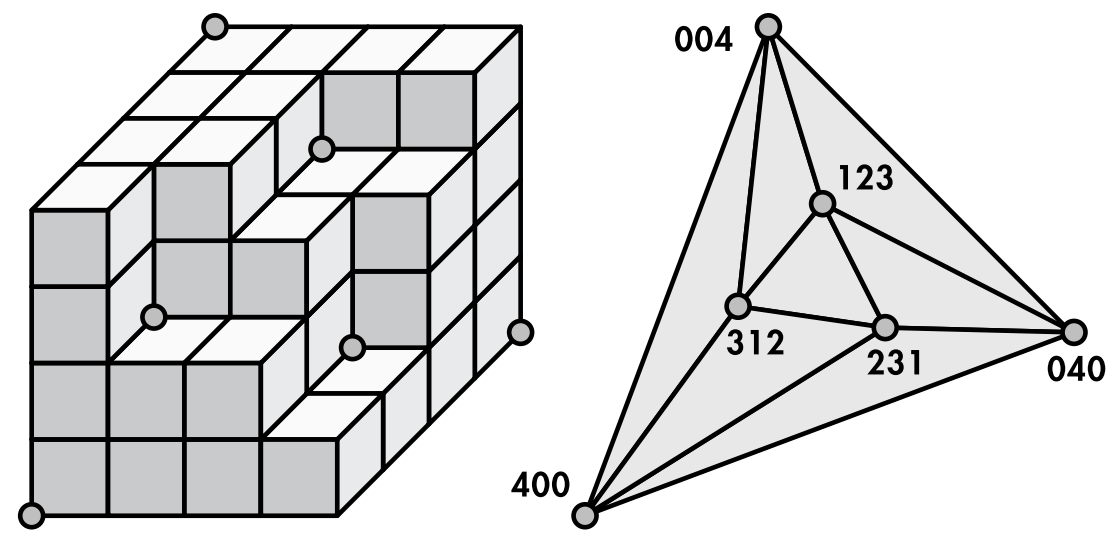

Note that $P_{M}$ is an octahedron. The minimal free resolution of $S / M$ equals $\mathbb{F}_{\Delta_{M}}$.

Proof of Theorem 5.1. Let $I$ be a face of $P_{M}$ and let $\mathcal{N}(I)$ denote the inner normal cone of $P_{M}$ at $I$. By Proposition 5.3, the Scarf complex $\Delta_{M}$ consists of all faces $I$ such that $\mathcal{N}(I)$ intersects the open positive orthant. Choose an $\epsilon>0$ such that $I \in \Delta_{M}$ if and only if the closed cone $\overline{\mathcal{N}(I)}=\bigcap_{i \in I} \overline{\mathcal{N}(\{i\})}$ intersects the $(n-1)$-simplex

$$
T:=\left\{\left(u_{1}, \ldots, u_{n}\right) \in \mathbb{R}^{n}: u_{1}+\cdots+u_{n}=1, u_{i} \geq \epsilon \text { for all } i\right\} .
$$

Then $\bigcap_{i \in I}(\overline{\mathcal{N}(i)} \cap T)$ is nonempty if and only if $I \in \Delta_{M}$. Thus $\{\overline{\mathcal{N}(i)} \cap$ $T\}_{1 \leq i \leq r}$ is a cover of $T$ by polytopes. The nerve of this cover equals $\Delta_{M}$. Using Borsuk's Nerve Lemma, we see that $\Delta_{M}$ is homotopy equivalent to $T$.

\section{Extremal combinatorics}

In this section we provide upper bounds for the Betti numbers of an arbitrary monomial ideal, and we explore further connections to extremal combinatorics. Set $\beta_{i}(n, r):=\max \left\{\beta_{i}(M)\right\}$ where the maximum is taken over all monomial ideals $M$ with $r$ minimal generators in $k\left[x_{1}, \ldots, x_{n}\right]$. It follows from Corollary 5.3 that $\beta_{i}(n, r)$ is attained by a monomial ideal $M$ which is generic. We may assume that $M$ is Artinian, by the following easy lemma: 
Lemma 6.1. Let $M=\left\langle m_{1}, m_{2}, \ldots, m_{r}\right\rangle$ be a generic monomial ideal where $m_{1}=x_{1}^{i_{1}} x_{2}^{i_{2}} \cdots x_{n}^{i_{n}}$ and $i_{1}>\operatorname{deg}_{x_{1}}\left(m_{j}\right)$ for $j \geq 2$. If $M^{\prime}=\left\langle x_{1}^{i_{1}}, m_{2}, \ldots, m_{r}\right\rangle$ then the Scarf complex $\Delta_{M}$ is a subcomplex of the Scarf complex $\Delta_{M^{\prime}}$.

Corollary 6.2. $\beta_{i}(n, r)$ equals the maximal number of $i$-faces of any Scarf complex $\Delta_{M}$, where $M$ runs over all Artinian generic monomial ideals $M$ with $r$ generators in $n$ variables.

Proof. Apply Lemma 6.1 repeatedly until all variables appear to some power. Take the resulting generic Artinian monomial ideal $M$ and apply Corollary 3.3 (1).

Each Scarf complex $\Delta_{M}$ considered in Corollary 6.2 is the boundary of a simplicial $n$-polytope with at least one facet removed, by Corollary 5.5 . The Upper Bound Theorem for Convex Polytopes (cf. [Zi, Thm. 8.23]) implies the following result:

Theorem 6.3. The Betti numbers of monomial ideals satisfy the inequalities of the Upper Bound Theorem for Convex Polytopes. More precisely, if $c_{i}(n, r)$ denotes the number of $i$-dimensional faces of the cyclic $n$-polytope with $r$ vertices, then

$$
\begin{aligned}
\beta_{i}(n, r) & \leq c_{i}(n, r) \quad \text { for } 1 \leq i \leq n-2, \\
\text { and } \quad \beta_{n-1}(n, r) & \leq c_{n-1}(n, r)-1 .
\end{aligned}
$$

An explicit formula for $c_{i}(n, r)$ is given in [Zi, §8]. For instance, $c_{1}(3, r)=$ $3 r-6, c_{2}(3, r)=2 r-4$ and $c_{1}(4, r)=\left(\begin{array}{l}r \\ 2\end{array}\right), c_{2}(4, r)=r(r-3), c_{3}(4, r)=$ $r(r-3) / 2$. For $n \leq 3$ all simplicial polytopes with $r$ vertices have the same $f$-vector, so the inequalities in Theorem 6.3 are equalities. The results in $[\mathrm{Ag}]$ show that the inequalities can be equalities for $n=4, r \leq 12$, but they are always strict inequalities for $n=4, r=13$. Agnarson proved that $\beta_{1}(4,13)=77$ while $c_{1}(4,13)=78$; this also implies $\beta_{2}(4,13)<c_{2}(4,13)$ and $\beta_{3}(4,13)<$ $c_{3}(4,13)-1$ by the Euler and Dehn-Sommerville equations.

We next relate Scarf complexes to the dimension theory of partially ordered sets (see $[\operatorname{Tr}])$. Recall that the order dimension $o \operatorname{dim}(\mathcal{P})$ of a finite poset $\mathcal{P}$ is the smallest number $s$ of linear extensions $L_{1}, \ldots, L_{s}$ of $\mathcal{P}$ such that $L_{1} \cap \cdots \cap L_{s}=$ $\mathcal{P}$. If $\Delta$ is a simplicial complex then $o \operatorname{dim}(\Delta)$ denotes the order dimension of its face poset. It is well-known that $o \operatorname{dim}(\Delta) \geq \operatorname{dim}(\Delta)+1$. The case of equality is of special interest for us:

\section{Theorem 6.4.}

(a) A simplicial complex $\Delta$ satisfies o $\operatorname{dim}(\Delta) \leq n$ if and only if $\Delta$ is a subcomplex of the Scarf complex $\Delta_{M}$ for some generic monomial ideal $M$ in $k\left[x_{1}, \ldots, x_{n}\right]$.

(b) Let $\Delta$ be a triangulation of the n-ball whose boundary equals the boundary of an $(n-1)$-simplex. Then $o \operatorname{dim}(\Delta)=n$ if and only if $\Delta$ equals the Scarf complex $\Delta_{M}$ of a generic Artinian monomial ideal $M$ in $k\left[x_{1}, \ldots, x_{n}\right]$. 
Proof. The if-direction in both (a) and (b) is seen as follows: For $i=1, \ldots, n$ let $L_{i}$ denote the linear extension of the face poset of $\Delta_{M}$ defined by $\Delta_{M} \rightarrow$ $\mathbb{R}, I \mapsto \operatorname{deg}_{x_{i}}\left(m_{I}\right)+\epsilon|I|$, where $\epsilon$ is a small positive real. The face poset of $\Delta_{M}$ coincides with $L_{1} \cap \cdots \cap L_{n}$.

We next prove the only-if direction in (a). Let $\Delta$ be a simplicial complex on $\{1, \ldots, r\}$ of order dimension at most $n$. Fix an embedding of posets $\phi: \Delta \rightarrow$ $\mathbb{N}^{n}$ such that each coordinate of $\phi$ is a linear extension of $\Delta$. We define the monomial ideal $M=\left\langle m_{1}, m_{2}, \ldots, m_{r}\right\rangle$ where $m_{i}:=\mathbf{x}^{\phi(\{i\})}$. Let $I$ be any face of $\Delta$. Note that $m_{I}=\operatorname{lcm}\left(m_{i}: i \in I\right)$ divides $\mathbf{x}^{\phi(I)}$. We must show that $I$ is a face of $\Delta_{M}$. Suppose not. Then there exists a subset $J$ of $\{1, \ldots, r\}$ with $I \neq J$ but $m_{I}=m_{J}$. If $J$ is not a subset of $I$ then pick any $j \in J \backslash I$. Then $m_{j}=\mathbf{x}^{\phi(j)}$ does not divide $\mathbf{x}^{\phi(I)}$ (since $\phi$ is a poset embedding), but $m_{j}$ does divide $m_{I}=m_{J}$, a contradiction. If $J$ is a subset of $I$ then $J$ is a proper face of $I$ in $\Delta$. In this case we pick any $i \in I \backslash J$. Now $m_{i}$ does not divide $\mathbf{x}^{\phi(J)}$ (since $\phi$ is a poset embedding), but $m_{i}$ does divide $m_{I}=m_{J}$, a contradiction.

We finally prove only-if in (b). By part (a) there is a generic monomial ideal $M$ such that $\Delta$ is a subcomplex of $\Delta_{M}$. We may assume that $\Delta$ and $\Delta_{M}$ have the same vertices. Applying Lemma 6.1 to the vertex labels of the $(n-1)$-simplex triangulated by $\Delta$, we may also assume that $M$ is Artinian. Both $\Delta$ and $\Delta_{M}$ are triangulations of the same $(n-1)$-simplex and $\Delta \subseteq \Delta_{M}$. Hence $\Delta=\Delta_{M}$.

Corollary 6.5. $\beta_{i-1}(n, r)$ equals the largest cardinality of a family $\mathcal{F}$ of $i$-sets in $\{1, \ldots, r\}$ such that the simplicial complex spanned by $\mathcal{F}$ has order dimension $n$.

It was shown in [Sp] (see also Theorem 2.13 in [Tr, $\S 7]$ ) that the complete graph on $r$ vertices has order dimension at least $2+\log _{2}\left(\log _{2}(r-1)\right)$. Therefore

$$
\beta_{1}(n, r) \leq\left(\begin{array}{l}
r \\
2
\end{array}\right)-1 \quad \text { for } r \gg n
$$

A central topic in discrete geometry is the Steinitz Problem of characterizing the face lattices of all convex polytopes. While this problem has a beautiful solution due to Steinitz in dimension 3 [Zi, Theorem 4.1], recent work of RichterGebert [Ri] shows that the Steinitz Problem for 4-dimensional polytopes is essentially equivalent to classifying all semialgebraic varieties (and hence intractible). We propose the following variant for monomial ideals:

(a) Classify all simplicial complexes which are Scarf complexes of generic (Artinian) monomial ideals with $r$ minimal generators in $k\left[x_{1}, \ldots, x_{n}\right]$.

(b) Classify all triangulations of the $(n-1)$-simplex whose face poset has order dimension $n$.

Theorem 6.4 implies that (a) and (b) are equivalent. This realizability problem is trivial for $n \leq 2$ variables. In three variables it is nontrivial but solved by a theorem of Schnyder (see $[\operatorname{Tr}]$ ): for any triangulation $\Delta$ of a triangle there 
exists a generic Artinian monomial ideal $M \subset k[x, y, z]$ such that $\Delta=\Delta_{M}$. The analogous result does not hold for $n=4$ :

Theorem 6.6. There exists a triangulation of the tetrahedron with seven vertices which is not the Scarf complex of any monomial ideal in four variables.

For the proof we need one lemma. Let $\Delta$ be any simplicial complex on $\{1, \ldots, n, \ldots, r\}$ which is a triangulation of the $(n-1)$-simplex $\{1, \ldots, n\}$. A labeling of $\Delta$ is a family of bijections $\left\{\phi_{I}: I \rightarrow\left\{x_{1}, \ldots, x_{n}\right\}\right\}_{I \text { facet of } \Delta}$ which satisfies the following two axioms:

(A) If $I$ is a facet of $\Delta$ and $i \in I \cap\{1, \ldots, n\}$ then $\phi_{I}(i)=x_{i}$.

(B) If $I$ and $J$ are facets of $\Delta$ which share a common ridge, i.e. $I \cap J$ has cardinality $n-1$, then $\left\{j \in I \cap J: \phi_{I}(j)=\phi_{J^{\prime}}(j)\right\}$ has cardinality $n-2$.

Lemma 6.7. The Scarf complex $\Delta_{M}$ of any generic artinian monomial ideal $M$ possesses a labeling $\phi^{M}$. We call the labelings of the form $\phi^{M}$ realizable.

Proof. The labeling $\phi^{M}=\left\{\phi_{I}^{M}\right\}_{I}$ facet of $\Delta_{M}$ is defined as follows: If $I$ is a facet of $\Delta_{M}$ and $j \in I$ then $\phi_{I}^{M}(j)$ is the unique variable missing in $m_{I} / m_{j}$. The map $\phi_{I}^{M}: I \rightarrow\left\{x_{1}, \ldots, x_{n}\right\}$ is a bijection, and it is straightforward to check the axioms $(\mathrm{A})$ and $(\mathrm{B})$.

Proof of Theorem 6.6. Consider the following triangulation of a tetrahedron 1234:

$\Delta=\{1237,1245,1256,1267,1347,1457,1567,2345,2356,2367,3456,3467,4567\}$.

The complex $\Delta$ is the boundary of the cyclic polytope $C_{4}(7)$ minus the facet 1234.

By Lemma 6.7 it suffices to prove that $\Delta$ does not admit any labeling. Suppose on the contrary that $\Delta$ possesses a labeling. Writing $a, b, c, d$ for the four variables, the following labels are forced by the axioms:

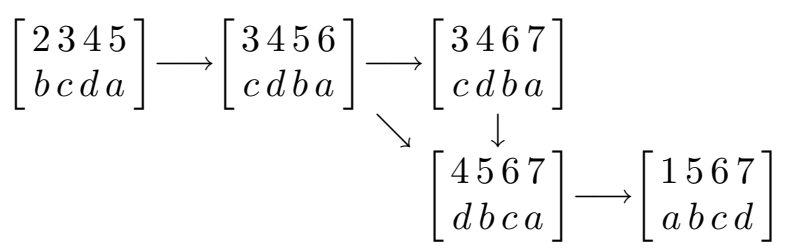

2345 is labeled by axiom (A), and 3456 and 3467 successively by axioms (A) and the "ridge axiom" (B). These last two tetrahedra each allow two labelings of 4567; the one shown is the common allowable labeling, forcing the labeling shown on 1567 .

On the other hand, the following labels are also forced:

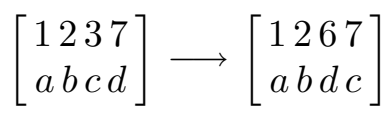

Now, there is a contradiction to axiom (B) in the labelings of 1267 and 1567. 


\section{Acknowledgements}

We are grateful to H. Scarf for inspiring discussions during his visit to Berkeley in April 1996; they got us started on this project. Many thanks to G. Ziegler

for helpful suggestions on Section 6 and to D. Eisenbud for suggestions on the exposition.

\section{References}

[Ag] G. Agnarsson, On the number of outside corners of monomial ideals, J. Pure Appl. Alg. 117 (1997), 3-22.

[Av] L. Avramov, Obstructions to the existence of multiplicative structures on minimal free resolutions, Amer. J. Math. 103 (1981), 1-31.

[BH] W. Bruns and J. Herzog, Semigroup rings and simplicial complexes, preprint, J. Pure Appl. Alg. (to appear).

[BLSWZ] A. Björner, M. Las Vergnas, B. Sturmfels, N. White, and G. Ziegler, Oriented matroids, Cambridge University Press.

[BW] A. Björner and M. Wachs, A. Björner and M. Wachs: Shellable nonpure complexes and posets, I., Trans. Amer. Math. Soc. 348 (1996), 1299-1327.

[Ei] D. Eisenbud, Introduction to commutative algebra with a view towards algebraic geometry (1995), Springer Verlag, New York.

[Ri] J. Richter-Gebert, Realization spaces of polytopes, Lecture Notes in Mathematics, 1643, Springer, Berlin, 1996.

[Ho] M. Hochster, Cohen-Macaulay rings, combinatorics and simplicial complexes, Ring Theory II (B. R. McDonald and R. Morris, eds.), pp. 171-223; Lecture Notes in Pure and Appl. Math., 26, Dekker, New York, 1977.

[Ku] A. Kustin, The minimal resolution of a codimension four almost complete intersection is a DG-algebra, J. Algebra 168 (1994), 371-399.

[Ly] G. Lyubeznik, A new explicit finite free resolution of ideals generated by monomials in an R-sequence, J. Pure Appl. Alg. 51 (1988), 193-195.

[PS] I. Peeva and B. Sturmfels, Generic lattice ideals, J. Amer. Math. Soc. (to appear).

[Sc] H. Scarf, The computation of economic equilibria, Cowles Foundation Monograph, 24, Yale University Press, 1973.

[Sp] J. Spencer, Minimal scrambling sets of simple orders, Acta Math. Acad. Scient. Hungaricae 22 (1971), 349-353.

[St] R. Stanley, Combinatorics and commutative algebra, Birkhäuser, Boston, 1996.

[Tr] W. Trotter, Combinatorics and partially ordered sets. Dimension Theory, The Johns Hopkins University Press, Baltimore, 1992.

[Zi] G. Ziegler, Lectures on polytopes, Springer Verlag, New York, 1994.

Dept. of Math., Barnard College, Columbia Univ., New York, NY 10027, USA

E-mail address: bayer@math.columbia.edu

2-277 Dept. of Math., M. I. T., Cambridge, MA 02139, USA

E-mail address: irena@math.mit.edu

Dept. of Math., Univ. of California, Berkeley, CA 94720, USA

E-mail address: bernd@math.berkeley.edu 